\title{
An empirical analysis of the super-latent inhibition effect
}

\author{
L. G. DE LA CASA \\ University of Seville, Seville, Spain \\ and \\ R. E. LUBOW \\ Tel Aviv University, Ramat-Aviv, Israel
}

\begin{abstract}
In three conditioned taste aversion experiments, we examined the roles of several variables in producing super-latent inhibition (LI). This effect, greater LI after a long interval than after a short interval between the conditioning and the test stages (De la Casa \& Lubow, 2000), was shown to increase with the number of stimulus preexposures $(0,2$, or 4; Experiment 1$)$ and with the length of the delay interval (1, 7, 14, or 21 days; Experiment 2). Furthermore, super-LI was obtained when the delay interval was introduced between the conditioning and the test stages (Experiments 1 and 2), but not when it was introduced between the preexposure and the conditioning stages (Experiment 3 ). The results are discussed in relation to interference explanations of LI.
\end{abstract}

Latent inhibition(LI) is defined as retarded performance on a learning task in which the target stimulus was previously irrelevant, as compared with cases in which it was not present. LI is most often explained by referring to some process, operative during the preexposure phase, that affects the subsequent associability of the stimulus (Lubow, Weiner, \& Schnur, 1981; Mackintosh, 1975; Pearce \& Hall, 1980; Wagner, 1981). Opposed to this acquisition failure consequence of unreinforced stimulus preexposure is the suggestion that LI is a result of retrieval failure (e.g., Bouton, 1993; Miller, Kasprow, \& Schachtman, 1986). As applied to three-stage (preexposure, conditioning, and test) LI procedures, such as conditioned taste aversion (CTA) and conditioned suppression, the retrieval failure hypothesis proposes that, following stimulus preexposure, the acquisition of the new association to the old stimulus proceeds normally. However, in the test stage, when the animal again encounters the target stimulus, two competing associations are retrieved, the earlier stimulus-no-consequence association from the preexposure stage and the stimulusUS association from the conditioning stage. The group that was not preexposed to the stimulus exhibits better learning than does the preexposed group, because there is only the second association to be retrieved. The stimulus-

This research was supported by DGES (Spanish government) Grant BSO2000-0323-C02-01. We thank Florencio Rengel for his help in conducting the experiments. Correspondence concerning this article should be addressed either to L. G. De la Casa, Department of Experimental Psychology, C/Camilo José Cela, s/n. 41005 Seville, Spain (e-mail: delacasa@us.es) or to R. E. Lubow, Department of Psychology, Tel Aviv University, Ramat-Aviv 69978, Israel (e-mail: lubow@ freud. tau.ac.il). preexposed group exhibits relatively poorer learning, either because the first association is the one that is retrieved (e.g., Kraemer \& Roberts, 1984) or because the first association interferes with the retrieval of the second one (Bouton, 1993).

Acquisition failure and retrieval failure theories of LI are based on the premise that something is learned during the preexposure period. They differ in terms of the locus of action of such learning. In the former case, the association that is learned in preexposure interferes with the acquisition of a new association. In the latter case, such acquisition is unaffected by stimulus preexposure. Instead, the association that was learned in the preexposure phase and the association that was learned in the acquisition phase compete for expression in the test phase.

The principal evidence for the retrieval/interference hypothesis of LI comes from two sources: studies in which it is demonstrated that postacquisition reminder treatments disrupt the conditioned stimulus (CS) preexposure effect (e.g., Kasprow, Catterson, Schachtman, \& Miller, 1984) and studies in which the time between the conditioning and the test stages has been varied. In regard to the latter, a number of CTA studies have reported that the LI that is present after a short delay is attenuated after a long delay (Aguado, de Brugada, \& Hall, 2001, Experiment 2; Aguado, Symonds, \& Hall, 1994, Experiment 1; Bakner, Strohen, Nordeen, \& Riccio, 1991, Experiments 1 and 2; De la Casa \& Lubow, 1995, Experiment 2). When this difference comes from the preexposed-long-delay group's drinking less of the preexposed and conditioned flavor than does the preexposed-short-delay group, this indicates that, with the short delay, the CS-unconditioned stimulus (US) association was present but not manifest. 
Something occurred during the longer delay that allowed the originally encoded, but not yet overt, CS-US association to be retrieved.

However, such an effect of delay has not been obtained consistently. Kraemer and Spear (1992) and Álvarez and López (1995, Experiment 2) failed to find LI attenuation after a long delay interval. More strikingly, De la Casa and Lubow (2000) have reported results that are diametrically opposite to that of LI attenuation with long delays. In three experiments, they found that LI was greater after a 21-day conditioning-test interval than after a 1-day interval

A review of the CTA literature on LI and delay reveals several procedural differences that may account for the apparently contradictory results. As has been noted by De la Casa and Lubow (2000) and Killcross (2001), experiments reporting delay-induced attenuation of LI have been conducted in the animals' home cages (e.g., Aguado et al., 1994). Conversely, experiments obtaining intact LI (Álvarez \& López, 1995) or super-LI used preexposure, conditioning, and test contexts that were different from the delay context. De la Casa and Lubow (2000) obtained the super-LI effect only when the preexposure, conditioning, and test stages were conducted in an apparatus other than the home cage whereas the delay period itself was spent in the home cage (Experiments 1, 2, and 3A). On the other hand, when all the experimental stages, including the delay period, took place in the home cages, there was no superLI (Experiment 3B). It would appear that, when the delay context is different from the context in which the CS and US are presented, the context-CS-nothing associations are protected from extinction and that the preservation of this association, perhaps enhanced by a primacy effect, is responsible for the super-LI effect.

In addition to context factors, there are other parametric differences between CTA experiments that have obtained super-LI and those that have obtained attenuated LI. In experiments in which attenuated LI has been obtained with delay, the number and length of flavor preexposures has ranged from six 15-min preexposures (Bakner et al., 1991) to only one 30-min preexposure (Kraemer \& Ossenkopp, 1986). Similarly, there have been wide variations in the length of the retention interval; attenuation of LI has been demonstrated with delays of 11 days (Bakner et al., 1991), 12 and 15 days (Aguado et al., 2001; Aguado et al., 1994) and 21 days (Kraemer \& Roberts, 1984). Thus, whereas attenuated LI has been demonstrated across a variety of parametric values, comparable data is lacking for the super-LI effect.

The present experiments were designed to identify the source of the super-LI effect in CTA. To this end, we used factorial designs to test the effects of number of preexposures and length of delay on LI (Experiments 1 and 2, respectively). The third experiment was designed to determine whether super-LI is dependent on the conditioningtest interval, as used by De la Casa and Lubow (2000), or whether it can also be generated by introducing a delay between the preexposure and the conditioning stages.

\section{EXPERIMENT 1}

It is well known that number of preexposures determines LI magnitude, at least when it is assessed after a relatively short interval. In three-stage LI-CTA preparations, the interval between the CS-US conditioning stage and the test stage is typically 1 or 2 days (see Lubow, 1989, for a review). However, there is no study of the effect of number of preexposures when a long delay is interpolated between the conditioning and the testing stages.

The first experiment in this series, then, was conducted in order to replicate the super-LI effect reported by De la Casa and Lubow (2000) and to examine the role of number of stimulus preexposures. To this end, we used a $2 \times$ $3 \times 3$ design, with two levels of delay between acquisition and test (1 and 21 days), three levels of flavor preexposure $(0,2$, and 4 days $)$, and three extinction trials in the test stage. If we accept a retrieval failure explanation of the super-LI effect, the strength of the first-learned association should modulate the expression of LI at the time of delayed testing. Specifically, the hypotheticalCS-nothing association established during preexposure would be stronger as the number of preexposures increases. Thus, we predicted not only that we would obtain a super-LI effect with the long acquisition-test interval (spent in a different context), thereby replicating De la Casa and Lubow (2000), but also that the size of the effect would be a function of the number of preexposures. Specifically, we expected an interaction between number of preexposures and delay, so that a super-LI effect would appear in the $4 \mathrm{PE} / 21$-day delay condition, but not in the 2PE/21-day delay condition.

\section{Method}

Subjects. The subjects were 48 adult naive male Wistar rats with a mean weight of $393.4 \mathrm{~g}$ (range, $356-473 \mathrm{~g} ; n=8$ per group). The animals were individually housed in $45 \times 20 \times 24 \mathrm{~cm}$ Plexiglas cages on a 12:12-h light:dark cycle. All testing occurred during the 12-h light period. Standard rat food was continuously available.

Apparatus. Experimental sessions were conducted in an isolated room, different from the colony room, where the subjects were housed during the delay intervals. The animals were transported to the experimental room, and each one was introduced into a $30 \times 18 \times$ $18 \mathrm{~cm}$ Plexiglas cage, where preexposure, conditioning, and test trials were conducted. Except for size, the experimental and the home cages were the same. The home cages were $43 \times 26 \times 15 \mathrm{~cm}$. However, the delay and the experimental environments were quite different from each other, including position of cages in rooms, orientation of light, smell, and so forth.

All liquid rations were provided at room temperature in 150-ml graduated plastic bottles, fitted with stainless steel spouts, similar to those used in the home cages. The bottles were attached to the front of each cage during liquid presentations. Amount of liquid intake was indexed by the difference between bottle weight before and after liquid presentation. The preexposure, conditioning, and test flavor was a $0.04 \%$ sodium saccharin solution. The unconditioned stimulus was a $0.5 \%$ of body weight intraperitoneal (i.p.) injection of $0.4-\mathrm{M} \mathrm{LiCl}$. This relatively high dosage was used because super-LI appears to be a positive function of US intensity. De la Casa and Lubow (2000) obtained the super-LI effect with 0.4 - and $0.5-\mathrm{M} \mathrm{LiCl}(0.5 \%$ of body weight), but not with $0.1-\mathrm{M} \mathrm{LiCl}(0.5 \%$ of body weight $)$. 
Procedure. After 7 days on a 23.5-h water deprivation schedule, maintained throughout the experiment except where indicated, the animals were matched for body weight and were assigned to six groups, each with 8 subjects. The stimulus preexposure stage was conducted on Days 8-11. All the sessions for each group lasted 5 min. ${ }^{1}$ For all the sessions (preexposure, conditioning, and testing), each animal was in the experimental cage for approximately $1 \mathrm{~min}$ preceding the 5-min drinking period. The animal was removed from the apparatus as soon as the $5 \mathrm{~min}$ had elapsed. There was a $24-\mathrm{h}$ interval between all the experimental sessions (except for the delay period in the 21-day condition). Two groups (NPE/1-day and NPE/21-days) received daily access to water. Another two groups received water on the first 2 days and saccharin on the last 2 days (2PE/1-day and 2PE/21-days). The remaining two groups (4PE/1day and 4PE/21-days) were preexposed to the saccharin solution on each of the 4 days. After each preexposure session, the animals were given access to water for $25 \mathrm{~min}$.

Conditioning was conducted on Day 12. All the animals received access to the saccharin solution for $5 \mathrm{~min}$, followed immediately by i.p. injections of LiCl. Groups in the 1-day delay condition were tested on Day 13; groups in the 21-day delay condition were tested on Day 32. In each one of the three test sessions, the subjects had 5-min access to the saccharin solution. Saccharin consumption was recorded on conditioning and test days. Each animal had access to water for 25 min after conditioning and test sessions.

To equate deprivation levels for all the subjects at the time of testing, water was available ad lib on Days 12-20 for those animals in the 21-day delay condition. The water deprivation schedule was reinstated on Day 21.

\section{Results and Discussion}

On the conditioning day, the mean amount of saccharin consumed by the NPE groups was $11.62 \mathrm{ml}(S D=1.09)$. The 2PE and 4PE groups consumed $11.7 \mathrm{ml}(S D=1.21)$ and $12.32 \mathrm{ml}(S D=1.98)$, respectively. The differences were not significant $[F(2,45)=1.08, p>.30]$.

Mean amounts of saccharin solution consumed in the test phase, combined for the three test trials, as a function of number of preexposures and delay are presented in Figure 1. As can be seen, there was a clear LI effect: The 2PE and 4PE groups exhibited less CTA (i.e., drank more) than did the NPE groups. This was true for the 1- and the 21-day delay conditions. However, the magnitude of LI (preexposure minus no preexposure) was a function of the number of preexposures and the length of delay between the acquisition and the test stages. Specifically, as compared with the 1-day delay condition, 21 days of delay produced a super-LI effect in the 4PE condition, but not in the $2 \mathrm{PE}$ condition. These observations were confirmed by a $3 \times 3 \times 2$ analysis of covariance (ANCOVA), with trials (within subjects), number of preexposures $(0,2$, and 4 ) and delay (1 and 21 days) as main factors and amount of saccharin solution consumed on the conditioning day as a covariate. There was a significant main effect of trials $[F(2,84)=128.64, p<.001]$, owing to progressive extinction of CTA across test trials, and a significant trials $\times$ number of preexposures interaction $[F(4,84)=$ $10.99, p<.001)$. The interaction was due to faster extinction as a function of number of preexposures, with subjects preexposed to saccharin drinking more across trials than did nonpreexposed subjects. The between- subjects analyses revealed significant main effects of number of preexposures and delay $[F(2,41)=62.00, p<$ .001 , and $F(1,41)=11.23, p<.01$, respectively]. Importantly, the preexposure $\times$ delay interaction also was significant $[F(2,41)=7.59, p<.01]$. Pairwise comparisons among the groups, using Duncan's tests $(p<.05)$, revealed that, for the 1-day condition, the $2 \mathrm{PE}$ and $4 \mathrm{PE}$ groups drank significantly more than did the NPE group. The same was true for the 21-day condition. In addition, Group 4PE/1-day consumed significantly less saccharin than did Group 4PE/21-days, revealing the expected superLI effect. On the other hand, Groups 2PE/1-day and 2PE/21-days did not differ in consumption of saccharin. In short, as was predicted, the long-delay-induced superLI effect was evident only in the group with the largest number of flavor preexposures. These data are congruent with the failure of De la Casa and Lubow (1995) to find a super-LI effect. In that study, the saccharin solution was preexposed for only 2 days, 5 min per day. There was no retention interval effect (e.g., Batsell \& Best, 1992) that is, conditioned aversion in the NPE/21-day group was not greater than that in the NPE/1-day group.

In both the present experiment and De la Casa and Lubow (1995), two preexposures produced robust LI in the 1-day interval condition. Nevertheless, in both cases, two preexposures were not sufficient to yield super-LI when the longer delay was introduced. This anomaly is not easily explained by reference to ceiling effects since, in the present experiment, the 4PE/21-day delay group consumed larger amounts of the saccharin solution than did the other groups.

\section{EXPERIMENT 2}

Experiment 2 was designed so that we could examine the role of length of delay between the acquisition and the test phases and, at the same time, to provide another replication of the basic super-LI effect. For this purpose, we used a $2 \times 4 \times 3$ design with two levels of stimulus preexposure $(0$ and 4$)$, four levels of delay between the acquisition and the test stages (1, 7, 14, and 21 days), and three extinction trials in the test stage. As in Experiment 1, we predicted an interaction - in the present case, between stimulus preexposure and length of delay. If the super-LI effect is promoted by longer delays, the magnitude of LI should increase as a function of delay length.

\section{Method}

Subjects and Apparatus. The subjects were 64 male Wistar rats ( $n=8$ per group). The mean weight was $422 \mathrm{~g}$ (range, 334-511 g). The animals were housed and fed as in Experiment 1.

Procedure. The procedure was identical to that in Experiment 1, except that all the PE groups received 4 days of flavor preexposure. Furthermore, a 7- and a 14-day delay group were added to the 1and 21-day delay groups of Experiment 1. In order to obtain an equivalent deprivation level for all the subjects at test day, the animals in the 14- and 21-day groups were given water ad lib on Days 12-14 and 12-20, respectively. However, for the 7-day group the deprivation schedule was maintained throughout the experiment. ${ }^{2}$ 


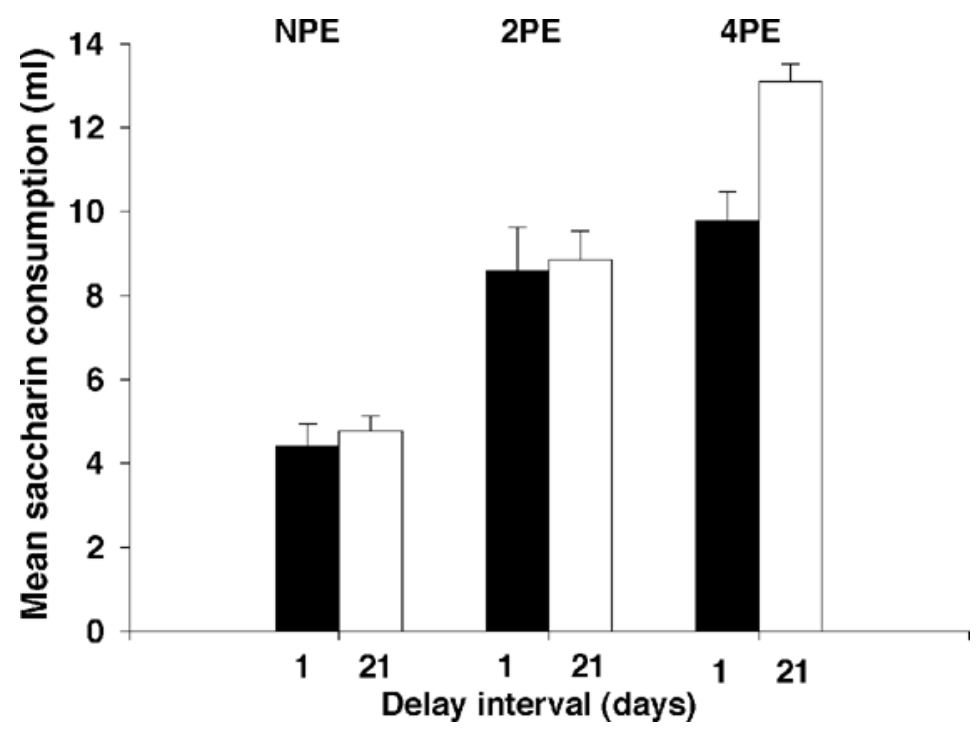

Figure 1. Mean amounts of saccharin solution consumed in the test stage (collapsed across the three extinction trials) as a function of amount of flavor preexposure $(0,2$, and 4 days) and delay between conditioning and test stages (1 and 21 days). Error bars represent $S E M$ s. NPE, no-preexposure group; 2PE, two-preexposure groups; 4PE, four-preexposure group.

Thus, at the time of test, the 7-day group had spent more days on the water deprivation schedule than had the other groups.

\section{Results and Discussion}

The mean amounts of saccharin solution consumed on the conditioning day for the NPE and the PE groups were $9.47 \mathrm{ml}(S D=2.14)$ and $8.58 \mathrm{ml}(S D=2.46)$, respectively, a nonsignificant difference $[F(1,62)=2.39, p>$ $.12]$. As in Experiment 1, subsequent analyses used the amount of saccharin consumed during test trials as an index of conditioning.

Figure 2 shows mean amounts of saccharin solution consumed in the test phase, combined for the three test trials, as a function of preexposure and delay interval. As can be seen, there was a clear LI effect for all PE versus NPE comparisons. However, the magnitude of LI (preexposure minus no preexposure) was a function of the length of delay between the acquisition and the test stages. Thus, a super-LI effect was produced with the 21-day delay, as compared with the 1-day delay. The 7- and 14-day delays did not affect LI magnitude. These observations were confirmed by a $3 \times 4 \times 2$ ANCOVA performed on amount of saccharin ingested in the test stage. The main factors were delay between the acquisition and the test stages $(1,7,14$, and 21 days), preexposure (0 and 4$)$, and trials. The amount of saccharin ingested on the conditioning day was used as a covariate. As was expected, there was a main effect of trials $[F(2,112)=57.72, p<.001]$ and a significant trials $\times$ preexposure interaction $[F(2,112)=5.84$, $p<.01]$. The trials $\times$ days of delay interaction was also significant $[F(6,112)=3.84, p<.01]$, owing to slower extinction with the intermediate levels of delay (7 and 14 days), as compared with 1 and 21 days. The three-way interaction was nonsignificant $[F(6,112)=1.93, p>$ $.08]$. The between-subjects analyses revealed main effects of delay and preexposure $[F(3,55)=6.16, p<.01$, and $F(1,55)=123.07, p<.001$, respectively]. Importantly, the delay $\times$ preexposure interaction was significant $[F(3,55)=8.04, p<.001]$. The interaction is shown in Figure 2, where the mean amount of saccharin is averaged for all extinction days and displayed as a function of number of preexposures and duration of the delay between conditioning and testing. As can be seen, the delay generated a super-LI effect only for the PE/21-day group. Pairwise comparisons using Duncan tests confirmed this observation. The LI effect for all PE versus NPE comparisons was significant ( $p$ s $<.05$ ). However, there was no significant difference between NPE groups or between PE/1-day versus PE/7-day versus PE/14-day groups $(p s>.05)$.

In summary, the super-LI effect was restricted to the longest conditioning-test delay interval. It is interesting to note that, as in Experiment 1, there was no retention interval effect in any of the NPE delay groups. In addition, although the data from the PE/7-day and PE/14-day groups tended to show a reduction in saccharin consumption over test days, the differences between them were nonsignificant. Thus, neither 7 nor 14 days of delay affected LI magnitude.

\section{EXPERIMENT 3}

Experiments 1 and 2 replicated the super-LI effect described by De la Casa and Lubow (2000). In addition, Ex- 


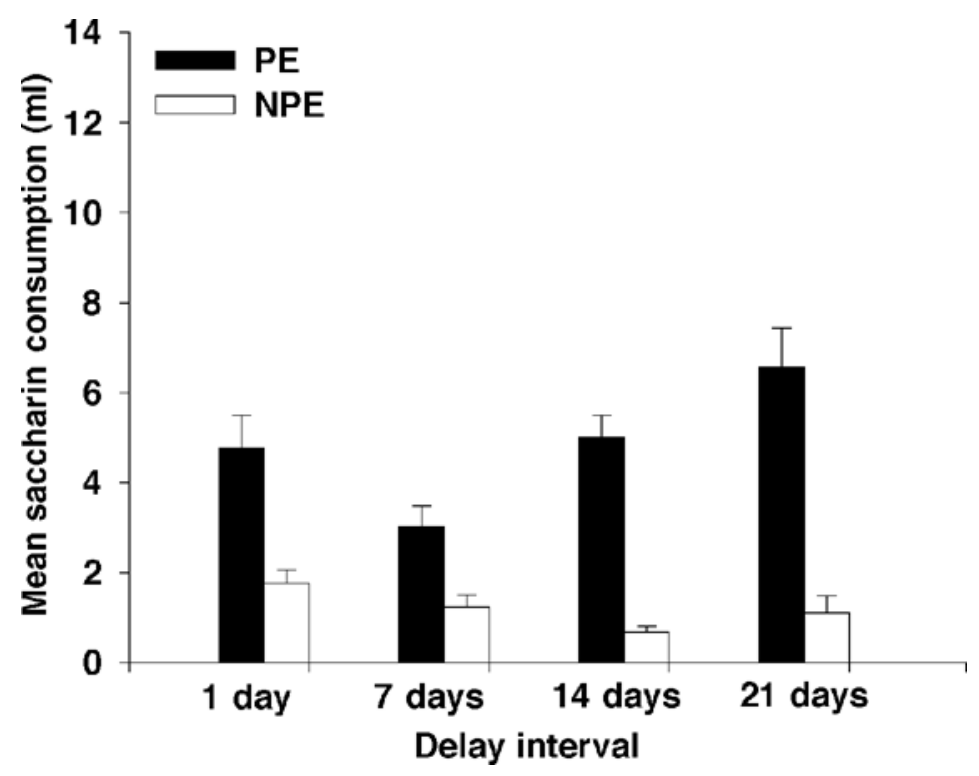

Figure 2. Mean amounts of saccharin solution consumed in the test stage (collapsed across the three extinction trials) by preexposed (PE) and nonpreexposed (NPE) groups as a function of delay between the conditioning and the test stages $(1,7,14$, and 21 days). Error bars represent $S E M$ s.

periment 2 demonstrated that the super-LI effect requires a relatively long delay between the conditioning and the testing phases. However, it has not yet been determined whether the effects of the long delay are dependent on its position between the conditioning and the test stages, a procedure used by all of the studies that have obtained super-LI. On the other hand, Aguado et al. (1994, Experiment 3 ) found LI attenuation after a 14-day delay between preexposure and conditioning. However, all the stages of the experiment, including the delay interval, were conducted in the same context. Since that appears to be a necessary condition for producing attenuated LI, as opposed to super-LI, it is important to determine whether the procedures that have proved to be successful in generating super-LI will produce similar effects when applied to the interval between preexposure and conditioning. A dissociation of preexposure-conditioning and conditioning-test interval effects has a direct bearing on explanations of super-LI. Specifically, the absence of super-LI when a long interval is introduced between the preexposure and the conditioning stages suggests (with the usual caveat about proving the null hypothesis) that the effect might best be considered as reflecting a retrieval competition process operating on associations learned in the preexposure and conditioning stages (e.g., Bouton, 1993; Kraemer \& Roberts, 1984; Miller et al., 1986), rather than as a preexposurebased process leading to acquisition failure in the conditioning stage (Lubow, 1989; Mackintosh, 1975; Pearce \& Hall, 1980; Wagner, 1981).

In the present experiment, we used a $2 \times 2 \times 3$ factorial design, with two levels of flavor preexposure $(0$ and 4 ) and delay interval between preexposure and condi- tioning phases ( 1 and 21 days) and with three extinction trials in the test stage. We have proposed that super-LI is produced by enhanced proactive interference (PI) that is generated when both CS-nothing and CS-US associations have been learned a relatively long time before the stage in which they are assessed (Lubow \& De la Casa, 2002). Thus, for the present experiment, we predicted that the LI effect would remain intact despite the 21-day delay between preexposure and conditioning, because the delay between the conditioning and the test was only 1 day. In fact, because the new conditions strongly favored the retrieval of the CS-US association, as opposed to the CSnothing association, simply as a result of contributions from recency, one might even expect attenuated LI in the 21-day delay group, as compared with the other groups without this recency advantage.

\section{Method}

Subjects. The subjects were 24 adult male Wistar rats $(n=6$ per group). The mean weight was $414 \mathrm{~g}$ (range 364-462 g). The animals were housed and maintained as described in Experiment 1.

Apparatus and Procedure. The apparatus and procedure were the same as those described for groups with zero and four flavor preexposures in Experiment 1, except that the delay was placed between the preexposure and the conditioning stages. To equate deprivation levels between groups at the time of conditioning and testing, the subjects in the 21-day delay condition had access to water ad lib on Days 11-19. The water deprivation schedule was reinstated on Day 20.

\section{Results and Discussion}

The mean amounts of saccharin solution ingested by the NPE and the PE groups on the conditioning day were 
$9.03 \mathrm{ml}(S D=3.02)$ and $10.51 \mathrm{ml}(S D=2.73)$, a nonsignificant difference $[F(1,22)=1.58, p>.22]$.

The mean amounts of saccharin solution consumed in the test phase, combined for the three test trials, as a function of preexposure and delay between preexposure and conditioning are presented in Figure 3. Again, we obtained a clear LI effect for both delay conditions. However, in contrast to previous experiments, the magnitude of LI was intact after 21 days of delay. These observations were confirmed by a $3 \times 2 \times 2$ ANCOVA performed on the amount of saccharin solution consumed on test trials. The main factors were preexposure (no preexposure vs. four preexposures), delay between conditioning and testing (1 vs. 21 days), and trials. Saccharin consumption on the conditioning day was used as a covariate. As in the previous experiments, the main effect of trials and the trials $\times$ preexposure interaction were significant $[F(2,40)=$ $25.33, p<.001$, and $F(2,40)=3.27, p<.05$, respectively]. None of the other interactions with trials was significant $(p s>.11)$. The between-subjects analyses revealed an effect of preexposure $[F(1,19)=20.28, p<$ $.001]$ Neither the effect of delay nor the interaction was significant $(p s>.25)$. As can be seen in Figure 3, which displays the mean saccharin consumption collapsed across the three test trials as a function of preexposure and delay, there was a robust LI effect. Moreover, the CS preexposure effect was not affected by the length of the delay period between preexposure and conditioning.

\section{GENERAL DISCUSSION}

The present set of three-stage CTA experiments demonstrated super-LI effects with a long delay (21 days) be-

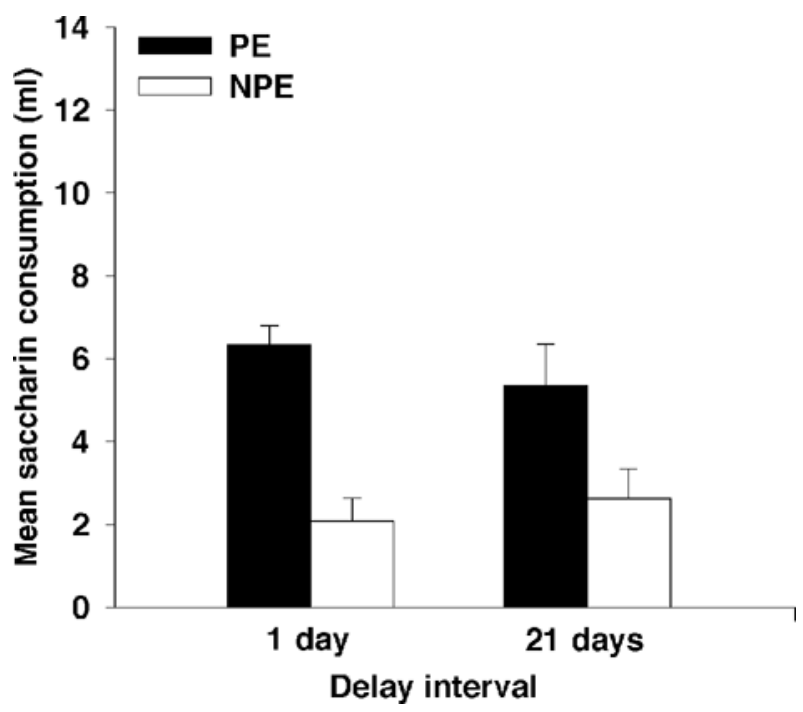

Figure 3. Mean amounts of saccharin solution consumed in the test stage (collapsed across the three extinction trials) by preexposed (PE) and nonpreexposed (NPE) groups as a function of delay between the preexposure and the conditioning stages ( 1 and 21 days). Error bars represent SEMs. tween the conditioning and the testing phases. In Experiment 1 , we found an enhanced LI effect when the animals were exposed four times to the CS, but not when exposed two times. Experiment 1 also indicated that normal LI, produced with a 1-day delay, was not sufficient for generating super-LI with a long delay. Thus, there was robust 1-day LI with two preexposures, but super-LI only with four preexposures. Experiment 2 revealed that super-LI was induced by long delays ( 21 days), but not by shorter ones ( 7 or 14 days). Finally, in Experiment 3, the delay between the preexposure and the conditioning stages (as opposed to the conditioning and the test stages) did not produce super-LI. This conclusion is, of course, tentative, since it is based on a null result, and it does not take into account differences in other time intervals created by the pre- and postconditioning interval manipulations. Furthermore, the absence of differences in the amount of LI in the short- and long-delay groups, as obtained in the present study, with the preexposure-acquisition delay periods spent in a different context from the remaining experimental stages, is in contrast to other CTA studies that have reported delay-induced attenuated LI when all stages were conducted in the home cage (e.g., Aguado et al., 1994, Experiment 3; Elkins \& Hobbs, 1979; McIntosh \& Tarpy, 1977). Nevertheless, the differences between the effects of pre-and postconditioning delays in the present experiment suggest that super-LI is largely the result of processing that occurs after the preexposure and conditioning manipulations.

This pattern of results fits more easily with a competition/interference explanation of LI (e.g., Bouton, 1993; Miller et al., 1986) than with traditional associative theories of LI, which do not address the effects of time. Specifically, Bouton (1993) has suggested that CS-alone preexposures generate a CS-nothing association that, at the time of testing, competes with the CS-US association acquired during conditioning. From this perspective, LI attenuation after delayed testing (e.g., Aguado et al., 2001; Aguado et al., 1994) would be the result of a recovery of a strong CS-US association after the delay, as compared with the interference produced by the CS-nothing association when the delay is short. However, it is not clear why the passage of time should favor the recovery of the CSUS association, as opposed to the CS-nothing association. The present results and those of De la Casa and $\mathrm{Lu}-$ bow (2000)—namely, the super-LI effect—highlight this problem.

An integrative explanation of the effects of delay on LI should take into account the factors that differentiate the hypothetical CS-nothing association from the CS-US association. First, in an LI experiment, the CS-nothing association is established before the CS-US association. Thus, normal LI may be thought of as reflecting a PI process (Bouton, 1993). Indeed, there is evidence that PI increases over time (e.g., Bouton \& Peck, 1992; Spear, 1978). Consequently, the CS-nothing association would be the source of more interference with the retrieval of the 
CS-US association after a long delay than after a short delay, resulting in the observed super-LI effect. The results of Experiment 1 support this view. When the CSnothing association was strengthened by increasing the number of stimulus preexposures, there was a concomittant increase in LI.Thus, two CS-alone preexposures did not strengthen the hypothetical CS-nothing association sufficiently to produce the super-LI effect after the 21day delay. However, with four stimulus preexposures, the super-LI effect was obtained, suggesting that the CSnothing association gained expression over time. That LI magnitude increased with the number of preexposures is congruent with the data from a large number of studies with short delays between stages (for reviews, see Lubow, 1973, 1989).

Interestingly, De la Casa and Lubow (2000, Experiments 1 and 2) provided evidence that super-LI requires a relatively strong US in the conditioning stage. The fact that the two manipulations - more stimulus preexposures and more intense US - that normally would be considered to strengthen competing associations have similar effects in the present preparation suggests that super-LI effects benefit from treatments that increase the discriminability between the flavor-no-consequence association and the flavor-US association. Note that this discriminability is independent of the discriminability of the two contexts in which they were acquired, since it is well documented that such differences in context have the opposite effect from that described above- that is, they disrupt LI (e.g., Lovibond, Preston, \& Mackintosh, 1984; Westbrook, Jones, Bailey, \& Harris, 2000; for a review, see Lubow, 1989, pp. 74-82).

In Experiment 2, super-LI was obtained, once again, with a 21-day interval between conditioning and test stages. However, the size of the LI effect with 7- and 14day intervals was no different from that of the 1-day interval. Whether one could obtain an even greater superLI effect with retention intervals longer than 21 days remains to be determined. Although it would be reasonable to expect such a result, particularly with an increase in the number of stimulus preexposures (see above), the possible potentiation may be obscured by ceiling effects.

In Experiments 1 and 2 and in the relevant studies with which they have been compared, the retention interval was operationally defined as the time between conditioning and test stages in a three-stage procedure. Consequently, whatever interval effects were uncovered, they are potentially attributable to factors related either to one or to both of the preceding stages (preexposure and conditioning). There are a number of other studies in which the time intervals between stages have been varied. However, the delay procedures and/or relationships among preexposure, conditioning, and test contexts differed from the ones used in the present experiments (e.g., Otto, Cousens, \& Rajewski, 1997; Rosas \& Bouton, 1997; Westbrook et al., 2000), thereby precluding any meaningful comparisons.

In Experiment 3, we examined LI with a new temporal relationship between preexposure, conditioning, and test stages. Specifically, in all the experiments showing superLI (Experiments 1 and 2 and those of De la Casa \& Lubow, 2000), the delay interval was placed between the conditioning and the testing stages. Consequently, the delay manipulations could have an approximately equal impact on the manner in which preexposure stage associations and conditioning stage associations affected test stage performance. In Experiment 3, unlike in our other experiments, we placed the delay interval between the preexposure and the conditioning phases. Under these conditions, a superLI effect was not obtained. However, we did find a "normal" LI effect even under conditions that should have favored retrieval of the CS-US association over the CSnothing association. The fact that LI survived a condition in which, for the 21-day delay PE group, the CS-US association was acquired 1 day before test, whereas the CSnothing association was acquired 21 days before test, again attests to the robustness of LI effects, particularly when the retention interval is spent in a context that is different from the preex posure, conditioning, and test stages. This latter point is supported by studies in which it was found that delays between preexposure and conditioning, when spent in the same context as the other stages of the experiment, resulted in attenuated LI (e.g., Aguado et al., 1994, Experiment 3).

Although it was not a main objective of our work, we obtained data relevant to the retention interval effectnamely, greater taste aversion when the test was conducted after a long rather than short conditioning-test interval (e.g., Batsell \& Best, 1992, 1994; Batsell \& George, 1996). The retention interval effect was not obtained in the present set of experiments or in those reported by De la Casa and Lubow (2000). That is, the NPE groups did not exhibit more conditioned aversion when tested after intermediate (7 and 14 days, Experiment 2) or long (21 days, Experiments 1,2, and 3) delays than when tested after a 1day delay. This discrepancy in results might be accounted for by differences in procedures. Positive demonstrations of the retention interval effect have been obtained with all experimental stages being conducted in the animals' home cages, thereby maintaining the same context throughout the entire experiment, including the delay.

In general, the use of home cages to conduct CTA experiments introduces a number of problems/confoundings. First, the home cage represents a safe environment that may be protected from the acquisition of the usual context-US associations that are common when experimental treatments are conducted in a distinctive new context (e.g., Archer, Sjoden, \& Nilsson, 1985). In this regard, Cole, VanTilburg, Burch-Vernon, and Riccio (1996) demonstrated that a CTA-based US preexposure effect appeared only when the experimental treatments took place in a new context, different from home cages. They concluded that the context-US associations presumably responsible for US preexposure effects were acquired when the context was novel, but not when it was familiar. Thus, pairing a new flavor with the US in the home cage may result in a stronger flavor-US association than does 
presenting flavor-US trials in a new context, since in the latter case the context would compete with the CS for acquiring associative strength (Rescorla \& Wagner, 1972). In addition, associative strength acquired to the home cage context would be extinguished during the long delay, since the animal has been exposed to the contextual cues without aversive consequences. Thus, after a long delay, at the time of test the conditioned flavor in the safe/ extinguished context would produce maximal CR expression. This view, albeit speculative, not only fits well with the absence of potentiated CTA in the NPE groups of the present experiments, but also may account for the attenuation of LI after delayed testing when such an effect is obtained with delay intervals spent in the home cage (e.g., Aguado et al., 2001; Aguado et al., 1994).

Finally, the super-LI effect and the proposed explanation may be specific to the CTA paradigm, at least insofar as the account refers to the acquisition of CS-alone (CS-no-consequence) associations during the stimulus exposure phase. Explanations of LI effects in CTA experiments, in general, have disregarded the fact that, with flavor preexposure, there is a consequence-namely, thirst reduction. Therefore, the competition in the test phase may be between the CS flavor that was reinforced positively (thirst reduction) and the same CS flavor that was reinforced negatively (LiCl-induced illness). This suggests that any generalizations from our data should be verified by similar experiments, but with different paradigms (e.g., conditioned suppression and/or appetitive procedures).

However, the validity of any theoretical analysis of LI effects rests on correctly identifying the associations that are learned in the preexposure and acquisition phases. Although there is agreement that the organism, in the acquisition phase, acquires a CS-US association and, perhaps, other associations as well, particularly to context, no such consensus exists for the preexposure phase. CS-noconsequence, CS-context, context-no-consequence, or a higher order conditional association whereby the context becomes an occasion-setter for the expression of the CS-no-consequence association are all possible candidates. It is not at all evident which, if any, of these possibilities is more or less compatible with acquisition failure or retrieval/competition interpretations of LI. What is unmistakably clear is that whether one obtains delay-induced attenuated LI or super-LI is critically dependent on the context of the delay interval. Such a finding provides a challenge to all extant learning theories.

\section{REFERENCES}

Aguado, L., De Brugada, I., \& Hall, G. (2001). Tests for inhibition after extinction of a conditioned stimulus in the flavour aversion procedure. Quarterly Journal of Experimental Psychology, 54B, 201-217.

Aguado, L., Symonds, M., \& Hall, G. (1994). Interval between preexposure and test determines the magnitude of latent inhibition: Implications for an interference account. Animal Learning \& Behavior, 22, 188-194.

Álvarez, R., \& LÓPEZ, M. (1995). Effects of elements or compound preexposure on conditioned taste aversion as a function of retention interval. Animal Learning \& Behavior, 23, 391-399.
Archer, T., SJoden, P., \& Nilsson, L. (1985). Contextual control of taste-aversion conditioning and extinction. In P. D. Balsam \& A. Tomie (Eds.), Context and learning (pp. 225-271). Hillsdale, NJ: Erlbaum.

Bakner, L., Strohen, K., Nordeen, M., \& Riccio, D. C. (1991). Postconditioning recovery from the latent inhibition effect in conditioned taste aversion. Physiology \& Behavior, 50, 1269-1272.

BATSELl, W. R., JR., \& Best, M. R. (1992). Variations in the retention of taste aversions: Evidence for retrieval competition. Animal Learning \& Behavior, 20, 146-159.

BATSELL, W. R., JR., \& Best, M. R. (1994). The role of US novelty in retention interval effect in single-element taste-aversion learning. Animal Learning \& Behavior, 22, 332-340.

Batsell, W. R., JR., \& George, J. W. (1996). Unconditioned stimulus intensity and retention interval effects. Physiology \& Behavior, 6, 1463-1467.

Bouton, M. E. (1993). Context, time, and memory retrieval in the interference paradigms of Pavlovian learning. Psychological Bulletin, 114, 80-99.

Bouton, M. E., \& Peck, C. A. (1992). Spontaneous recovery in crossmotivational transfer (counterconditioning). Animal Learning \& Behavior, 20, 313-321.

Cole, K. C., VanTilburg, D., Burch-Vernon, A., \& Riccio, D. C. (1996). The importance of context in the US preexposure effect in CTA: Novel versus latently inhibited contextual stimuli. Learning \& Motivation, 27, 362-374.

De la Casa, L. G., \& Lubow, R. E (1995). Latent inhibition in conditioned taste aversion: The roles of stimulus frequency and duration, and amount of fluid ingested during preexposure. Neurobiologyof Learning \& Memory, 64, 125-132.

De la CASA, L. G., \& Lubow, R. E. (2000). Super-latent inhibition with delayed conditioned taste aversion testing. Animal Learning \& Behavior, 28, 389-399.

Elkins, R. L., \& Новвs, S. H. (1979). Forgetting preconditioning CS familiarization and taste aversion learning: An animal experiment with implications for alcoholism treatment. Behaviour Research Therapy, 17, 567-573.

Kasprow, W. J., Catterson, D., Schachtman, T. R., \& Miller, R. R. (1984). Attenuation of latent inhibition by postacquisition reminder. Quarterly Journal of Experimental Psychology, 36B, 53-63.

KILlCROSS, S. (2001). Loss of latent inhibition in conditioned taste aversion following exposure to a novel flavour before test. Quarterly Journal of Experimental Psychology, 54B, 271-288.

Kraemer, P. J., \& Ossenkopp, K. P. (1986). The effects of flavor preexposure and test interval on conditioned taste aversions in rats. Bulletin of the Psychonomic Society, 24, 219-221.

Kraemer, P. J., \& Roberts, W. A. (1984). The influence of flavor preexposure and test interval on conditioned taste aversion in the rat. Learning \& Motivation, 15, 259-278.

Kraemer,P. J., \& Spear, N. E. (1992). The effect of nonreinforced stimulus exposure on the strength of a conditioned taste aversion as a function of retention interval: Do latent inhibition and extinction involve a shared process? Animal Learning \& Behavior, 20, 1-7.

Lovibond, P. F., Preston, G. C., \& Mackintosh, N. J. (1984). Context specificity of conditioning, extinction, and latent inhibition. Journal of Experimental Psychology: Animal Behavior Processes, 10, 360-375.

Lubow, R. E. (1973). Latent inhibition. Psychological Bulletin, 79, 398-407.

LuBow, R. E. (1989). Latent inhibition and conditioned attention theory. New York: Cambridge University Press.

Lubow, R. E., \& DE la CASA, L. G. (2002). Super-latent inhibition and spontaneous recovery: Differential effects of pre-and post-conditioning CS-alone presentations after long delays in different contexts. Manuscript submitted for publication.

Lubow, R. E., Weiner, I., \& Schnur, P. (1981). Conditioned attention theory. In G. H. Bower (Ed.), The psychology of learning and motivation (Vol. 15, pp. 1-49). New York: Academic Press.

Mackintosh, N. J. (1975). A theory of attention: Variations in the associability of stimuli with reinforcement. Psychological Review, 82, 276-298.

McIntosh, S. M., \& TARPY, R. M. (1977). Retention of latent inhibition in a taste aversion paradigm. Bulletin of the Psychonomic Society, $\mathbf{9}$ 411-412. 
Miller, R. R., Kasprow, W. J., \& Schachtman, T. R. (1986). Retrieval variability: Sources and consequences. American Journal of Psychology, 99, 145-218.

Otto, T., Cousens, G., \& Rajewski, K. (1997). Odor-guided fear conditioning in rats: Acquisition, retention, and latent inhibition. Behavioral Neuroscience, 111, 1257-1264.

Pearce, J. M., \& Hall, G. (1980). A model for Pavlovian conditioning: Variations in the effectiveness of conditioned but not unconditioned stimuli. Psychological Review, 87, 332-352.

Rescorla, R. A., \& Wagner, A. R. (1972). A theory of Pavlovian conditioning: Variations in the effectiveness of reinforcement and nonreinforcement. In A. H. Black \& W. F. Prokasy (Eds.), Classical conditioning II: Current research and theory (pp. 64-99). New York: Appleton-Century-Crofts.

Rosas, J. M., \& Bouton, M. E (1997). Additivity of the effects of retention interval and context change on latent inhibition: Toward resolution of the context forgetting paradox. Journal of Experimental Psychology: Animal Behavior Processes, 23, 283-294.

SPEAR, N. E. (1978). The processing of memories: Forgetting and retention. Hillsdale, NJ: Erlbaum.

WAGNER, A. R. (1981). SOP: A model of automatic memory processing in animal behavior. In N. E. Spear \& R. R. Miller (Eds.), Information processing in animals: Memory mechanisms (pp. 5-47). Hillsdale, NJ: Erlbaum.
Westbrook, R. F., Jones, M. L., Bailey, G. K., \& Harris, J. A. (2000). Contextual control over conditioned responding in a latent inhibition paradigm. Journal of Experimental Psychology: Animal Behavior Processes, 26, 157-173.

\section{NOTES}

1. Pilot studies conducted in our laboratory have demonstrated that with the deprivation level of the present experiments, animals consumed in 5 min approximately $80 \%$ of the fluid that they normally would consume in $30 \mathrm{~min}$.

2. The animals in the 1-day delay condition were on the water deprivation schedule for 13 days when they began the test stage. The animals in the 14- and 21-day delay groups were allowed free access to water from the day after conditioning until 13 days before testing. However, the 7-day delay group had only 7 days between conditioning and the first test day. Consequently, it was not possible to equate deprivation level at the test stage for this group with those for the remaining groups. Therefore, for this group, the deprivation level was maintained for the duration of the experiment.

(Manuscript received November 26, 2001; revision accepted for publication February 27, 2002.) 